\title{
DROGODEPENDENCIAS EN PERSONAL SANITARIO, UNA VISIÓN DESDE LA MEDICINA DEL TRABAJO (II). PROCEDIMIENTO DE REINCORPORACION LABORAL
}

\author{
CARMEN OTERO DORREGO*, CARMEN HUERTA CAMARERO**, NURIA DURO PERALES***
}

(*) Médico Especialista en Medicina del Trabajo y Técnico Superior en Prevención de Riesgos Laborales, Hospital de Móstoles (Móstoles, Madrid).

(**) Médico del Trabajo y Técnico Superior en Prevención de Riesgos Laborales.

(***) Técnico Superior en Prevención de Riesgos Laborales, Licenciada en Derecho,

Hospital de Móstoles (Móstoles, Madrid).

\begin{abstract}
Agradecimientos: a Dña. Concepción Lora Tamayo, Responsable del Departamento de Drogas del Instituto Nacional de Toxicología, al Dr. Carlos Mingote Adan, Responsable del PAIPSE, a la Dra. Ma Ángeles Sánchez Uríz, Coordinadora del SPRL del Área 1 de Madrid, al Dr. Luis Mazón Cuadrado, Coordinador del SPRL del Hospital de Fuenlabrada, por toda la colaboración e información suministrada a este SPRL.
\end{abstract}

\section{RESUMEN}

El seguimiento de la reincorporación laboral (de personal sanitario con drogodependencia), indispensable para evitar las recaídas, precisa una colaboración multidisciplinar (psicoterapeuta, médico del trabajo, etc) y un programa de control de drogas riguroso que consiste en realizar test -a intervalos aleatorios- intermitentes de drogas en muestras autentificadas de orina utilizando pruebas confirmatorias (cromatografía de gases junto con espectrofotometría de masas) al que se pueden añadir pruebas en muestras de pelo en cada escalón del seguimiento.

\section{PALABRAS CLAVES}

Médico del Trabajo, drogodependencias en personal sanitario, procedimiento de seguimiento de reincorporación laboral, pruebas confirmatorias.

\begin{abstract}
It concludes that monitoring the return to work (problems of substance abuse in health personnel), which is essential to avoid relapses, requires a multidisciplinary collaboration (psychotherapist, medical officer, etc.) and a drug control program is to conduct rigorous tests at random intervals intermittent of drugs in urine samples authenticated using confirmatory tests (along with gas chromatography and mass spectrometry) to which you can add tests on samples of hair in every stage of follow-up.
\end{abstract}

\section{KEY WORDS}

Medical labour, substance abuse in health personnel, procedure monitoring the return to work, confirmatory tests. 


\section{INTRODUCCION}

Los problemas de drogodependencias en personal sanitario suponen un importante reto para la responsabilidad profesional del médico del trabajo ${ }^{(1)}$ : debe abordar la rehabilitación laboral (y personal) del profesional sanitario drogodependiente tratado, evitando las recaídas, que pueden dar lugar a daños a terceros (daños a los pacientes, mala praxis) y/o a sí mismo (suicidios, p.ej.). Se establece el procedimiento de actuación (vigilancia de la salud obligatoria, contrato terapéutico, consentimiento informado) y se revisan los tipos de pruebas diagnósticas (base de datos MedLine) que puede utilizar el médico del trabajo para el seguimiento durante la reincorporación laboral del profesional sanitario drogodependiente (tras el tratamiento de su adicción). Dicho seguimiento se demuestra que es indispensable para evitar recaídas o minimizar los daños derivados de las mismas.

1. ¿CÓMO ABORDA EL MÉDICO DEL TRABAJO EL PROBLEMA DE PROFESIONALES SANITARIOS CON ADICCIONES Y DROGODEPENDENCIAS?

El profesional sanitario con un problema de adicción cumple cuatro condiciones:

a) Es un enfermo ${ }^{(2)}$ : el médico del trabajo deberá disponer a su disposición todos los métodos posibles de diagnóstico y tratamiento para su curación. Deberá hacer una previsión de una posible recaída.

b) Es un usuario del sistema de salud: el médico del trabajo deberá respetar sus derechos básicos (derecho al consentimiento informado, derecho a la debida reserva -secreto profesional-, y derecho a la firma de un contrato terapéutico, entre otros) ${ }^{(2,3,4)}$.

c) Es un compañero: el médico del trabajo, como cualquier otro facultativo, debe recordar el contenido del Art. 19 del Código de Ética y Deontología ${ }^{(5)}$ de la Organización Médica Colegial. Según dicho artículo, si un médico apreciase que un compañero no estuviera en las adecuadas condiciones para ejercer su trabajo, tiene la obligación de ponerlo en su conocimiento y pedirle que se deje tratar por algún médico de su confianza y además comunicarlo a su Colegio Oficial de Médicos, de forma objetiva y con la debida discreción, porque el bien de los pacientes está por encima de todo.

d) Es un trabajador sanitario: por tanto es competencia de su Servicio de Prevención de Riesgos Laborales (SPRL). El médico del trabajo, tras realizarle un examen de salud y evaluarle, deberá emitir un informe en términos de "aptitud" según su capacidad funcional para su puesto de trabajo (Art. 22 de la Ley 31/1995, de Prevención de Riesgos Laborales $(\mathrm{LPRL}))^{(6)}$. Todos los reconocimientos son voluntarios, salvo las tres excepciones que se citan en el Art. 22 de la LPRL: cuando sea imprescindible para evaluar los efectos de las condiciones de trabajo sobre la salud de los trabajadores, cuando sea necesario verificar si el estado de salud de un trabajador constituye un peligro para sí mismo o para terceras personas relacionadas con la empresa, cuando así esté establecido en alguna disposición legal en relación con la protección a los riesgos específicos y actividades de especial peligrosidad.

El objetivo del médico del trabajo es doble: conseguir la rehabilitación personal y laboral del "sanitario enfermo" de forma que se consiga la reincorporación laboral tras el tratamiento de desintoxicación y deshabituación, sin que el trabajador pueda ser causa de daños a terceros (sus propios pacientes), ni a sí mismo (suicidio, recaídas). Es una tarea complicada que requiere valorar el puesto de trabajo, el nivel de tareas de responsabilidad hacia los usuarios, y el tipo de trastorno adictivo que presente (por establecer un símil, un adicto a opiáceos trabajando en un servicio de anestesia, sería como un niño en una "tienda de golosinas" $\left.{ }^{(7)}\right)$.

Algunas situaciones anómalas pueden hacer sospechar a compañeros y responsables directos que un profesional sanitario padece un trastorno adictivo: trastornos del comportamiento, somnolencia importante, alteraciones físicas (pérdida de peso, sudoración profusa, miosis o midriasis, sangrados nasales frecuentes, etc), reclamaciones de los pacientes (p.ej. todos los pacientes de un mismo anestesista que se despiertan más pronto y con más dolor que el resto de pacientes del servicio, puede ser un indicio de que se 
les está poniendo menor dosis de fentanilo de la prescrita, guardándose una parte de las dosis para el propio consumo).En estos casos, siempre que se documente adecuadamente el problema, y ante la sospecha de actuaciones que ocasionen un riesgo para sí mismo o para terceros (los pacientes), los responsables del centro sanitario pueden solicitar al médico del trabajo que le realice un examen de salud o reconocimiento médico obligatorio en virtud del Art. 22 de la LPRL ${ }^{(6)}$. Según dicho articulo, como ya se ha mencionado anteriormente, la vigilancia de la salud será obligatoria en el caso de que un trabajador pueda representar un daño para sí mismo o para terceros, pero siempre "previo informe de los representantes de los trabajadores". En la práctica, no es viable presentar casos individuales en las reuniones del Comité de Seguridad y Salud para la aprobación por los miembros del Comité (representantes de los trabajadores y otros) por un problema ético y legal: la confidencialidad. Este trámite legal se puede solventar a través de dos cauces, contemplando el posible riesgo a terceros en la Evaluación Inicial de Riesgos de aquellos puestos con atención a pacientes (que, además, tendrá una graduación diferente según que la actividad del trabajador sea asistencial, o no), o bien, siguiendo las recomendaciones de la Dra. Sánchez Uríz, estableciendo un "procedimiento de vigilancia de la salud obligatoria (VSO)"(8) que se presenta una única vez al Comité de Seguridad y Salud para su aprobación y que contemple todos los supuestos que se pudiesen plantear, sin que ninguna de estas vías excluya a la otra.

Antes de continuar, conviene aclarar que no se puede establecer ningún sistema de control ajeno al reconocimiento médico laboral, que no esté debidamente solicitado y argumentado desde sus superiores jerárquicos con motivo de riesgos a sí mismo o a terceros, según se refiere en los dos puntos siguientes:

1. Todos los reconocimientos son voluntarios, salvo que se cumplan las condiciones del Art. 22 de la ley 31/1995 de Prevención de Riesgos Laborales

2. Principios rectores para realizar pruebas analíticas de alcohol y drogas en el trabajo. Noruega. Mayo. 1993. OIT, recomendaciones para la adopción de medidas e investigaciones: realizar la investigación a fin de evaluar la relación entre consumo de alcohol y drogas con la seguridad y productividad en el trabajo ${ }^{(9)}$.

\subsection{Procedimiento de Vigilancia de la Salud Obligatoria (VSO).}

El procedimiento, citando las pautas de Sánchez Uríz, debe partir de tres premisas iniciales:

a) Que ante sospecha de patología con riesgo para terceros y/o para si mismo de un trabajador, percibida por compañeros, responsables y/o representantes de los trabajadores se debe documentar dicha situación (descripción de las alteraciones del comportamiento, situaciones de riesgo o reclamaciones de los pacientes).

b) Que debe emitirse una solicitud escrita por parte de la Dirección dirigida al Servicio de Prevención (SPRL) sobre la "valoración médica, es decir, examen de salud, por sospecha de riesgo para terceros" aportando la documentación descrita en el apartado anterior.

c) Que una vez recibida la solicitud y la documentación en el SPRL, éste citará (por vía convencional) al trabajador, según el Art. 22 de la Ley 31/1995, para realizar examen de salud con las mismas garantías con las que realiza habitualmente la vigilancia de la salud a todos los trabajadores (confidencialidad, etc.).

A partir de este momento, el trabajador puede acudir o no, lo que dará lugar a las diferentes actuaciones del médico del trabajo del SPRL (figura 1), y

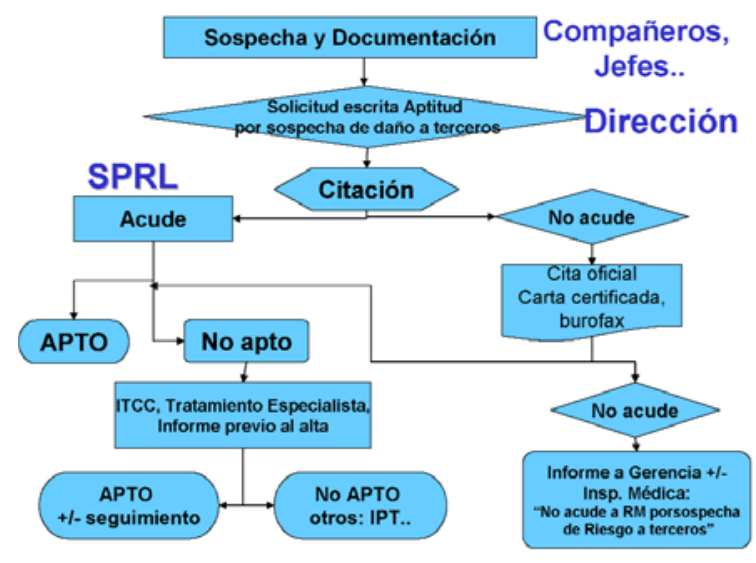

Figura 1. Procedimiento de Vigilancia de la Salud Obligatorio (VSO). 
que deben quedar descritas en el documento del procedimiento.

Cuando aparece un caso nuevo (figura 2) puede acudir de forma voluntaria al SPRL para pedir ayuda profesional, o, lo que es más frecuente, condicionado a un procedimiento de Vigilancia de la Salud

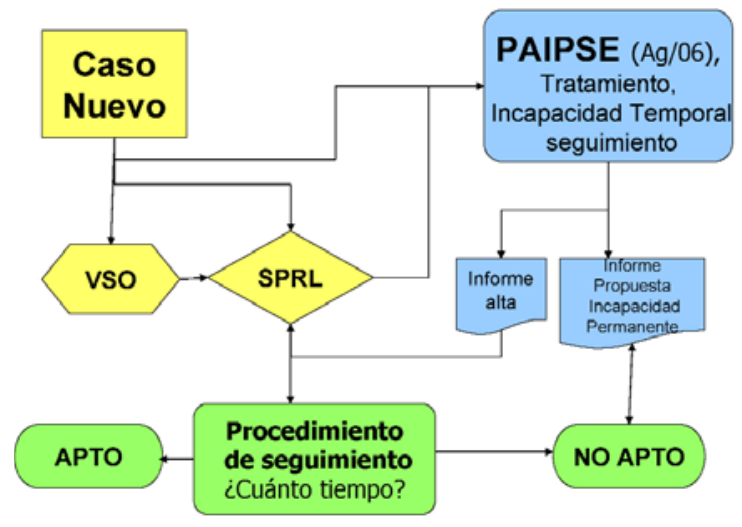

Figura 2. Caso nuevo

Obligatorio (VSO). El médico del trabajo del SPRL le evaluará (historia clínico-laboral: anamnesis detallada, exploración física, pruebas complementarias, riesgos laborales asociados al puesto según la evaluación de riesgos, etc.) y decidirá si precisa ayuda especializada (psiquiatra, psicoterapeuta). En este punto se hace necesario recordar que las historias clínico-laborales de los trabajadores deben encontrarse en un archivo propio de los SPRL, adecuadamente custodiadas, y bajo ningún concepto puede tener acceso a ellas nadie que no sea el propio personal del SPRL. Desde el SPRL se derivará al "sanitario enfermo" a un programa de ayuda integral (desde agosto 2006 esta operativo el PAIP$\mathrm{SE}^{(10)}$ en la Comunidad de Madrid) donde se le va a tratar (desintoxicación, deshabituación, periodo de incapacidad transitoria) cuándo es posible su reincorporación laboral (si no, directamente se le gestionaría un proceso de incapacidad permanente). También existe la posibilidad de que sea el "profesional enfermo" el que acuda directamente al programa de ayuda. En cualquier caso, su psicoterapeuta emitirá un informe de alta, con reincorporación laboral, o un informe propuesta de incapacidad permanente. Si se reincorpora al trabajo habitual, será el médico del trabajo del SPRL el que tenga que realizar el seguimiento, sobre todo para evitar recaídas que puedan dar lugar a una situación de daño para sí mismo (suicidios, p.ej.) o para terceros (los pacientes). Lo difícil es determinar cómo realizar ese seguimiento para que sea riguroso, con las pruebas toxicológicas más adecuadas, garantizando la colaboración del profesional sanitario en fase de deshabituación reincorporado a su trabajo, y cuanto tiempo debe durar dicho seguimiento.

\subsection{Procedimiento de seguimiento en fase de recuperación y rehabilitación profesional.}

El SPRL debe definir un procedimiento de seguimiento en la reincorporación laboral fundamentado en:

a) Un Contrato Terapéutico donde se establezcan las condiciones del seguimiento.

b) Un protocolo de recogida, conservación y envío de muestras (Controles Toxicológicos). Determinar los tipos de controles toxicológicos que se van a realizar y su indicación. Establecer el Laboratorio de Referencia.

Tener una previsión de las posibles recaídas. Valorar la duración más adecuada del seguimiento para evitar recaídas y tener prevista la actuación en caso de que se produzca.

Revisar el nivel de evidencia de las intervenciones realizadas.

\subsubsection{Contrato Terapéutico.}

"Es mediante un contrato terapéutico la forma en que los médicos enfermos se comprometen por escrito a comportarse como enfermos, a seguir los tratamientos farmacológicos y/o psicológicos (y seguimientos), a cumplir las restricciones necesarias en la práctica profesional, a someterse a los controles analíticos que se establezcan, etc". Arteman Jané(11).

Además, el derecho a la firma del contrato terapéutico se encuentra recogido en la normativa vigente (Ley de Drogodependencias) ${ }^{(2)}$.

El contenido mínimo de un contrato terapéutico debe abordar los siguientes apartados: 
a) Datos de Identidad del profesional sanitario al que se le va a realizar el seguimiento de reincorporación laboral.

b) Identidad del responsable (psicoterapeuta) del tratamiento de deshabituación y/o psicoterapia.

c) Arbitraje del SPRL para realizar controles en el lugar de trabajo y emitir Aptitud laboral.

d) Se le facilita una "falsa identidad" para preservar la intimidad (confidencialidad al realizar el envío de muestras al laboratorio, etc.).

e) Tipo de controles toxicológicos que se van a realizar.

f) Adaptación progresiva a la normalidad laboral y condiciones (asumir tareas de más complejidad y responsabilidad escalonadamente, p.ej. sin actividad de quirófano ni guardias, con actividad de quirófano tutelada $\mathrm{y}$ sin guardias, añadiendo medias guardias, etc. según se vayan cumpliendo periodos de tiempo preestablecidos con todos los controles negativos, en el caso de los anestesistas).

\section{Contrato Terapéutico: ACUERDO DE REHABILITACIÓN LABORAL EN FASE DE ABSTINENCIA}

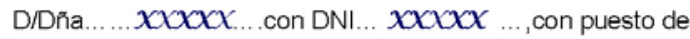
trabajo.................... Servicio ................., turno, .........se compromete con la Dirección/SPRL de este Centro Sanitario, mediante este escrito a:

1.-Designar como persona responsable de su tratamiento a D./Dña. en calidad de

\section{Psicoterapeuta}

2.-Acudir a revisión en el SPRL (que actuará como Servicio de arbitraje en cuanto a su aptitud laboral) cuando sea requerido y aportar documentación/informe periódico favorable de su psiquiatra de estar acudiendo a las revisiones, hasta cursar alta por curación en aras de favorecer su realización personal y laboral.

3.-Someterse a análisis de orina/sangre/pelo aleatorios (sin previo aviso) según marque el servicio de arbitraje (SPRL) SIEMPRE en presencia de personal sanitario del SPRL y contrapruebas más específicas en caso de positivos

4.-Comprometerse a no tomar medicación alguna que falsee el resultado de las pruebas, dando su consentimiento por escrito cada vez que se realice una prueba y explicitando en dicho consentimiento la medicación que toma en ese momento o la ausencia de medicación. Para preservar su intimidad la petición de las pruebas se hará bajo una falsa identidad que será la siguiente:... J. Pérez Pérez.................
Duración del proceso.

g) Condiciones en caso de "positivos" en los controles analíticos.

h) Posibilidad de negativa al tratamiento $\mathrm{y} / \mathrm{o}$ seguimiento, y consecuencias derivadas de dicha negativa.

i) Fecha y firmas (del profesional causante del contrato terapéutico y del responsable de la Dirección del Centro Sanitario y/o Responsable del SPRL, dependiendo de cómo se haya planteado).

Se adjunta un modelo posible de Contrato Terapéutico (figura 3 ).

\subsubsection{Protocolo para los controles toxicológicos.}

Antes de definir el protocolo de recogida, almacenaje y envío de muestra al laboratorio, es preciso determinar qué controles debemos realizar: el tipo de pruebas de laboratorio más indicadas, el tipo de muestras biológicas adecuadas para el seguimiento y los requisitos del laboratorio al que se le pide que analice las muestras que le enviemos.
-En caso de que los análisis sean positivos a algún tóxico, el paciente autoriza al Facultativo del SPRL a comunicar dichos resultados a sus responsables inmediatos (Jefe de Servicio, y Dirección del Centro), dado que no se puede aceptar el riesgo ocasionado a terceros (los pacientes). La positividad confirmada a cocaína y/o cannabis y/o opiáceos y/o anfetaminas, descartada la existencia de interferencias medicamentosas, será motivo de valoración como NO APTO.

- Tiene derecho a negarse a aceptar estas condiciones dado que le asiste el derecho a la protección de su intimidad, según la Ley Orgánica 15/1999, de 13 de diciembre de Protección de Datos de Carácter Personal. Pero en caso de una negativa, se estaría en un supuesto de duda razonable de que el riesgo sigue persistiendo y se interpretaría en los mismos términos que una prueba de toxicológica positiva, en dicho caso no se podría aceptar el riesgo ocasionado a los pacientes

- La recuperación de la normalidad laboral será progresiva incorporándose primero a la actividad $X \times X X X$ después de $X$ semanas de controles negativos, $y$ al menos $X$ semanas más de controles negativos para su incorporación a $x \times x \times x$

-La positividad de un control supone su paso a tareas de menor responsabilidad.

- La segunda positividad de un control en menos de $X$ tiempo supone la comunicación al Colegio Oficial de Médicos de...... y la inhabilitación colegial/profesional .

En ........., a...... de ......... de 200.

Fdo

Paciente-trabajador Responsable Dirección/SPRL 
a) Tipos de técnicas de análisis toxicológico. Las pruebas deben ser fiables.

Por las implicaciones legales se considera que no se deben pedir meras pruebas de screening (basadas en técnicas de inmunoanálisis), sino pruebas confirmatorias $^{(9)}$, siendo la GC (cromatografía de gases) conjuntamente con espectrofotometría de masas (GC-MS) la técnica considerada más adecuada y fiable en la actualidad ${ }^{(12,13)}$.

b) El laboratorio de elección también debe ser fiable.

En los Laboratorios de Bioquímica de los hospitales generales sólo se pueden realizar técnicas de screening (técnicas de inmunoanálisis) que no sirven para el seguimiento. Los controles toxicológicos se deben externalizar, enviando las muestras a un laboratorio que reúna todas las condiciones de calidad en técnicas de GC-MS y con amplia experiencia acreditada en el campo del análisis de drogas. En nuestro medio, nuestro SPRL ha considerado como laboratorio de referencia al Departamento de Drogas del Instituto Nacional de Toxicología y Ciencias Forenses. Las muestras enviadas son custodiadas durante tres meses desde que se emite el informe, con la posibilidad de solicitar, si se estima necesario, un contra-análisis.

c) Las muestras deben estar adecuadamente elegidas e indicadas.

Actualmente la orina es el fluido biológico preferido para el análisis de uso de drogas ilegales y sus metabolitos ${ }^{(12)}$, siempre que se garantice la autenticidad de las muestras para evitar los falsos negativos por su adulteración. Lo más relevante es la lectura de positivos en cuanto a los puntos de corte (Cut-off) y el tiempo de duración de la detección. Los laboratorios oficiales cuentan con una gran experiencia en estos estudios. Los tiempos de duración de detección no varían proporcionalmente con la dosis de la droga madre consumida, así se observa que una mayor dosis de droga consumida por un individuo, sólo prolonga ligeramente el tiempo de detección (p.ej. después de la aspiración nasal de 1,5 mg de cocaína puede detectarse benzoilecgonina en orina hasta dos o tres días después) $^{(14)}$.
Se ha notificado que la programación del análisis de orina 3 días por semana es la más eficaz para la detección del uso de heroína o cocaína, sin embargo genera un alto coste y sólo se recomienda en circunstancias excepcionales. Los test a intervalos aleatorios intermitentes pueden ser los que tengan mejor coste-eficacia ${ }^{(12)}$.

La recogida de sangre es probablemente el procedimiento menos favorable para el test rutinario de drogas, aunque es la muestra más útil para la identificación de drogas en análisis cuantitativos. Indicación: urgencias clínicas, identificación de consumo muy reciente de drogas (las sustancias psicoactivas que se analizan a menudo abandonan la sangre rápidamente, p.ej. la cocaína tiene una vida media de 1 hora en sangre). Se debería utilizar ante una urgencia, una vez realizado un screening en orina y/o como prueba confirmatoria. De todas formas, las concentraciones sanguíneas de cocaína deben interpretarse con prudencia dado que no predicen ni la gravedad de los hallazgos clínicos, ni la incidencia de los efectos adversos, ni el pronóstico o la necesidad de intervención terapéutica ${ }^{(14)}$. Este SPRL no considera adecuada esta prueba para el seguimiento (monitorización química) del adicto tratado que se reincorpora a la actividad laboral.

Desde la década de los 90 se viene proponiendo el pelo de cuero cabelludo como nuevo elemento biológico para el test de uso ilegal de dro$\operatorname{gas}^{(12,15,16,17)}$. Las ventajas descritas son la inexistencia de riesgo de falsificación por abstinencia temporal o adulteración (cuestionable, pueden no llegar a detectarse consumos puntuales o puede alterarse con tintes con peróxido), la recogida de muestra no invade la privacidad del individuo (también cuestionable -la toma de muestra deja una "calva" en el vertex bastante visible-), la posibilidad de tomar una segunda muestra de la misma persona para contraanálisis (salvo que se corte el cabello al ras) y la estabilidad de la muestra incluso bajo condiciones ambientales adversas (se puede almacenar sin refrigeración). No obstante el pelo debe ser visto como un complemento de otros procedimientos de despistaje de consumo de drogas o para establecer una historia de droga a lo largo del tiempo (consumos crónicos). La GC-MS es el mejor procedimiento de laboratorio para analizar drogas en el 
pelo $^{(12,13,15)}$, ya que satisface criterios de identificación y de cuantificación.

Sin embargo, están descritos bastantes problemas que dificultan su aplicación: el procedimiento de la toma de muestra de pelo (tabla I) no es un procedimiento simple ${ }^{(12,17)}$, la concentración de drogas fijadas en el cabello pigmentado es mucho mayor que

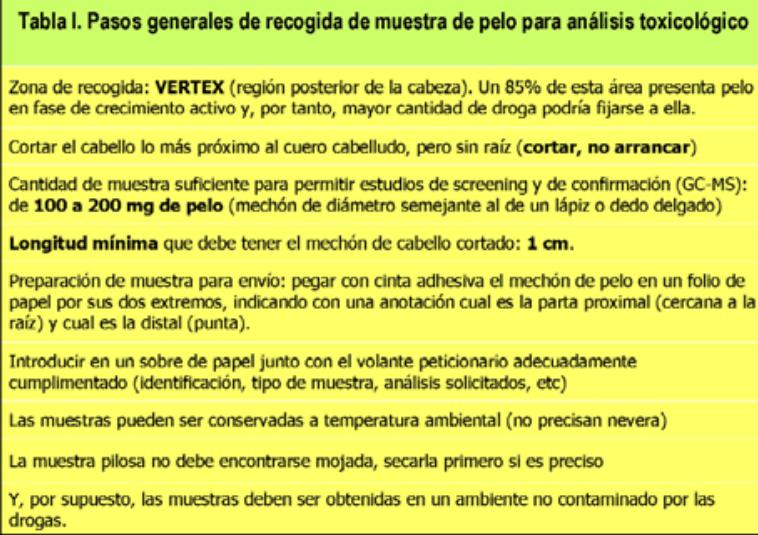

en cabello claro (la melanina juega un rol importante en la incorporación de drogas en el pelo $\left.{ }^{(12,17)}\right)$, los tratamientos capilares (peróxido blanqueador, etc.) pueden alterar los niveles de droga en el pelo ${ }^{(12)}$, existe irregularidad del crecimiento del cabello entre individuos e incluso en un mismo individuo ${ }^{(12,17)}$ por eso el cabello se toma universalmente de la parte posterior del vértex parieto-occipital que se cree menos susceptible a estas variaciones-, además, las propiedades fisicoquímicas de cada sustancia concreta juegan un papel en el Índice de Incorporación de la droga al pelo (IRC) (la cocaína tiene el IRC más alto y el cannabis el más bajo, así como las drogas ácidas, que son especialmente difíciles de detectar en el pelo del cuero cabelludo de humanos) $)^{(12)}, \mathrm{y}$, el peor supuesto de todos, la posibilidad de falsos positivos por contaminación de la cara externa del pelo por las concentraciones de drogas ambientales (riesgo de falsos positivos exógenos) ${ }^{(16,17)}$, dificultando la distinción de una administración voluntaria de la droga de una involuntaria (administración pasiva).

Gold y otros autores ${ }^{(18,19)}$ defienden un nuevo factor de riesgo, además de los clásicos ${ }^{(7)}$, asociado al consumo de opiáceos como sustancias de abuso entre los anestesistas: la exposición ambiental a aerosoles de opiáceos. Wolf mide en aire las concentraciones de opiáceos intravenosos (propofol y fentanilo) que se utilizan en pacientes y que se aerosolizan en el ambiente y comprueba unas altas concentraciones ambientales a las que los anestesistas están expuestos durante sus horas de trabajo. El problema que se nos plantea en nuestro caso, es si estas teóricas concentraciones ambientales pudiesen falsear pruebas cómo el análisis de pelo (falsos positivos por contaminación exógena).

Además, conviene conocer la experiencia que se tiene de la práctica de la técnica en nuestro medio: detección de drogas en pelo mediante cromatografía de gases-espectrofotometría de masas en los laboratorios a los que podemos acceder en nuestro medio (tabla II) y el coste de la prueba.

\begin{tabular}{|c|c|}
\hline \multicolumn{2}{|c|}{$\begin{array}{l}\text { Tabla II. Conocer las determinaciones de algunas drogas en el } \\
\text { laboratorio de referencia. Preguntar al laboratorio. } \\
\text { La técnica empleada es la cromatografia de gases-espectrometria de masas } \\
\text { (GC-MS) que es especifica para cada uno de los compuestos siguientes: }\end{array}$} \\
\hline \multirow{2}{*}{$\begin{array}{l}\text { BENZOILECGONINA } \\
\text { metabolito final de } \\
\text { cocaina (la cocaina } \\
\text { desaparece antes de la } \\
\text { orina): }\end{array}$} & $\begin{array}{l}\text { En orina ( para } 2 \mathrm{ml} \text { ) } \\
\text { Limite de detección: } 10 \text { nanogramos / ml } \\
\text { Limite de cuantificación: } 20 \text { nanogramos / ml }\end{array}$ \\
\hline & $\begin{array}{l}\text { En pelo (para } 40 \text { miligramos): Aparece cocaina y en } \\
\text { menor cantidad benzoilecgonina. Criterio. Solo se da } \\
\text { positivo si aparecen los dos. La cuantificación que se } \\
\text { hace es aproximada. } \\
\text { Limites de detección (aprox.):0,1 nanogramos } / \mathrm{mg} \\
\text { Limites de cuantificación (aprox.):0,3 nanogramos } / \mathrm{mg}\end{array}$ \\
\hline \multirow[b]{2}{*}{ FENTANILO: } & $\begin{array}{l}\text { En orina (para } 2 \mathrm{ml} \text { ) } \\
\text { Limite de detección: } 154 \text { nanogramos } / \mathrm{ml}\end{array}$ \\
\hline & $\begin{array}{l}\text { En pelo (para } 40 \mathrm{mg} \text { ), térico } 4 \text { nanogramosg/mg. La } \\
\text { posologia tan baja del Fentanilo, hace que los niveles } \\
\text { esperados en cabello sean de picogramos o } \\
\text { fentogramos a los que el limite de deteoción de la } \\
\text { técnica no alcanza. Por esta razón este compuesto no } \\
\text { se analiza habitualmente en muestras de pelo en este } \\
\text { Centro (Instituto Nacional de Toxicologia) }\end{array}$ \\
\hline
\end{tabular}

Por todos estos motivos, este SPRL tampoco ha considerado adecuada esta prueba para la monitorización química. Lo más adecuado en el seguimiento serían los test a intervalos aleatorios intermitentes de muestras (autentificadas) de orina.

d) Se debe establecer un protocolo de recogida, custodia y envío de muestra riguroso.

\section{d.1) Procedimiento de recogida y custodia de} la muestra:

El control toxicológico por el SPRL en el propio lugar de trabajo es más fiable que cualquier otro control que se pueda pedir a un drogodependiente dentro de los programas de retorno (acuden "con cita previa"), porque el trabajador no puede "prepararse" para intentar conseguir "falsos negativos" en caso de 
que siga consumiendo. Aún así, al recoger la muestra, se deben tener en cuenta las siguientes recomendaciones:

1.- Citación inmediata (llamada) en el SPRL. Si pone problemas (evitar que gane tiempo para beber "litros" de agua -dilución de la muestra de orina- y untarse las manos de jabón -película de jabón que "filtraría" la muestra de orina-): el/la enfermero/a del SPRL acude a su puesto de trabajo para la toma de muestra. En ocasiones, si el responsable directo (jefe de servicio, supervisor, etc) está implicado en el proceso de reincorporación laboral del "sanitario enfermo", se puede solicitar su colaboración para que consiga que alguien sustituya momentáneamente al trabajador al que se le hacen los controles y no pueda poner excusas para no acudir al SPRL inmediatamente (p.ej: "ahora mismo no puedo, tengo un paciente y tardaré como media hora").

2.- Consentimiento informado escrito antes de la recogida de muestra (ver figura 4, Modelos de

\section{D. / Dna........................ con clave identificativa} Juan Pérez Pérez..

En el día de la fecha he sido informado/a de que el análisis de orina $\mathrm{y} / \mathrm{o}$ sangre $\mathrm{y} / \mathrm{o}$ pelo que se me ha realizado hoy incluye la investigación de drogas de abuso (Opiáceos/Heroína, Cannabis/Marihuana, Cocaína y anfetaminas) y que el mismo cumple con los requisitos de control de calidad normalmente requeridos.

Otras determinaciones:

fentanilo, Benzodiacepinas,

Me doy por enterado y doy mi consentimiento a que se practique dicho análisis.

Para descartar posibles interferencias

medicamentosas, conteste a la siguiente pregunta:

¿Está tomado actualmente algún tipo de medicación, o la ha tomado en los últimos días?

$\square$ Sí ¿Cuáles?

$\square$ No

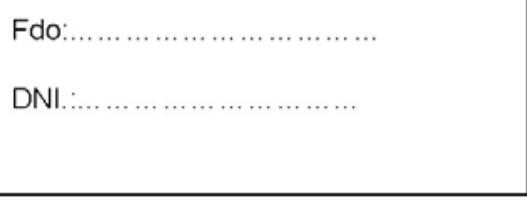

"consentimiento para realizar análisis") para cualquier tipo de muestra (excepción: no se precisaría consentimiento informado escrito en caso de urgencia vital por sospecha de sobredosis). Se cumplimenta por duplicado: se guarda una copia en la historia clínico laboral y se entrega otra copia al trabajador. Es muy importante que conste la fecha.

3.- Debe haber un testigo presencial de la toma de muestra (autentificación de la muestra) en el caso de muestras de orina: el enfermero/a del SPRL. No hace falta "doble muestra" para contraanálisis, dado que cuando la muestra llega al laboratorio toxicológico, una de sus garantías es la custodia de la misma muestra durante tres meses desde que emite su informe, para posibilitar la petición de un contraanálisis.

4.- Etiquetado por el enfermero/a del SPRL (código y nombre con la identidad falsa facilitada).

$$
\begin{aligned}
& \text { En............, a..... de ................ de } 200 . . \\
& \text { D. I Dña..................... con clave identificativa }
\end{aligned}
$$
......Juan Pérez Pérez........

En el día de hoy he sido informado del resultado del análisis de orína $y / o$ sangre $y \longdiv { / o ~ p e l o }$ que se me ha realizado el día......... de......................de 200 ... para la investigación de drogas de abuso.

Resultado negativo a:

Resultado positivo a:

En caso de positivo:

Ejerzo / Renuncio (táchese lo que no proceda) el/al derecho a que se practique un contraanálisis y elijo el Laboratorio que cumple con los requisitos de control de calidad normalmente requeridos y asumo los gastos que se deriven de la realización de esta prueba.

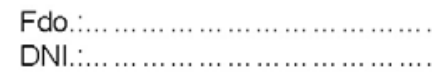

Figura 4. Modelos de "Consentimientos para hacer análisis" e "Informe de resultados" 
5.- Conservación en nevera (del propio SPRL) hasta envío. Las muestras se pueden conservar varios días en frío (al menos $48 \mathrm{~h}$ ), pero por las implicaciones legales que pueda tener la aparición de un "positivo", lo recomendable es que se envíen al laboratorio de referencia lo más rápidamente posible.

\section{d.2) Procedimiento de envío de la muestra:}

1.- Petición debidamente cumplimentada (por el facultativo del SPRL). Antes de iniciar este tipo de seguimiento, el médico del trabajo responsable del mismo debe ponerse en contacto con el responsable del laboratorio toxicológico al que va a enviar las muestras, explicando cómo se va a ir desarrollando todo el proceso y solicitando a su vez información de cómo se debe enviar cada tipo de muestra (orina, pelo, sangre). El laboratorio toxicológico suele tener un modelo de petición de pruebas propio que tiene que enviar al médico del trabajo antes de que éste solicite la primera analítica. Cuando se cumplimente dicho impreso, el nombre del trabajador será el que se le haya otorgado como "identidad falsa", y también se hará por duplicado. Una copia es la que se envía al laboratorio toxicológico, y la otra copia se adjunta a la del "consentimiento informado para realizar análisis", guardando ambas en la historia clínico-laboral del trabajador (en el archivo propio del SPRL), adecuadamente custodiada.

2.- Preparar dos sobres de papel: uno con la muestra (con adecuado embalaje para evitar extravasaciones) y otro con la petición, grapados ambos. Correctamente cerrados y con las señas del laboratorio toxicológico perfectamente identificadas (dirigido al responsable del departamento de drogas del laboratorio toxicológico, con el que se ha mantenido contacto previo).

3.- Envío con "salida por Registro" del Centro Sanitario.

4.- Transporte por celador (pactado previamente) o por los medios que se consideren adecuados (mensajería urgente, etc.). Se debe tener en cuenta tanto el coste del transporte, como el de las propias pruebas (gastos de laboratorio) a la hora de diseñar el procedimiento de seguimiento.

\subsubsection{Previsión de recaídas.}

Para establecer una pauta de actuación será adecuado tener conocimiento de la posibilidad de recaídas, a qué factores de riesgo se asocian y durante cuanto tiempo se suelen presentar. Se debe contemplar:

1) Que existe un índice de incidencia de recaída asociado al colectivo de anestesistas que se han reincorporado a su trabajo tras haberse sometido a tratamiento de deshabituación que puede variar desde un $\mathbf{1 9 \%}$ hasta un $\mathbf{4 0 \%}$ según la bibliografía consulta$\mathrm{da}^{(7,20,21,22,23)}$. Aunque hasta un $70 \%$ de los profesionales de salud pueden reincorporarse con éxito a su trabajo después del tratamiento, de los 22 anestesistas que consumieron fentanilo como droga de elección en el programa Washington Physicians Health Program (WPHP) sólo 5 pudieron retornar a su trabajo habitual sin ninguna recaída ${ }^{(20)}$.

2) Que en el análisis retrospectivo de los datos obtenidos del WPHP (desde 1/1/1991 hasta 31/12/2001), el riesgo de recaída (hazard risk, HR) fue casi el doble cuando interviene un opióide potente como droga de elección en comparación con otras sustancias de abuso (HR 1.80, IC 1.03-3.13, p = 0.04), o con comorbilidad (asociación de trastorno psicopatológico coexistente con la adicción) (HR 2.12, IC 1.33-3.36, p = 0.02), o con antecedentes familiares del consumo de drogas $(\text { HR 2.14, IC 1.18-3.90, } \mathrm{p}=0.01)^{(20)}$.

3) Que la prevalencia de recaídas del grupo profesionales de la salud (médicos de diferentes especialidades, dentistas, enfermeros, farmacéuticos...) en programas de monitoreo post-tratamiento fue de un $25 \%$ para una primera recaída, un $5 \%$ tuvieron dos recaídas y un $3 \%$ tuvieron tres o más recaídas. La mayoría de las recaídas se detectó por monitoreo químico (en el curso del programa de ayuda) y/o en el lugar de trabajo, y en un $58 \%$ de los casos se produjeron durante los dos primeros años en el programa. La estimación de las recaídas acumulativas a los 5 años fue del $35 \%$ para los opióides mayores en comparación con el $25 \%$ para otras drogas ${ }^{(20)}$. 
4) Que una posible recaída supone un riesgo para terceros (mala praxis, reclamaciones de los pacientes ${ }^{(7,24)}$ y un importante riesgo de daño para sí mismo, ya que se ha observado que la muerte puede ser la forma de presentación inicial de recaída en el $16 \%$ en el

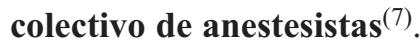

5) Que una posible recaída puede presentarse como una urgencia clínica (síntomas y signos sugerentes de consumo de sustancias de abuso) y en ese caso la prueba confirmatoria indicada sería una analítica de sangre urgente para detección de drogas y sustancias de abuso en ese momento.

6) Que el riesgo de recaída disminuye $(p<0.001)$ con el incremento en la duración del programa de seguimiento. La tasa de recaída se estimó en 91/1000 personas/año en los dos primeros años del programa, disminuyó a 58/1000 personas/año en los dos a cinco años de programa, y a 32/1000 personas/año después de los cinco años de progra$\mathrm{ma}^{(20)}$.

Según estos datos, se puede estimar que los programas de monitoreo que pretendan evitar o disminuir el riesgo de recaída de los profesionales sanitarios en proceso de recuperación de su adicción deben ser más intensos y prolongados en los sanitarios en los que confluyan varios factores de riesgo o que hayan presentado recaídas previas.

\subsubsection{Revisión del nivel de evidencia de las inter- venciones realizadas.}

En una revisión sistemática de las guías clínicas para la intervención en drogas realizada dentro del ámbito de la medicina de Atención Primaria ${ }^{(25)}$ se asociaron las diferentes actividades a realizar en la intervención en drogas con un determinado nivel de evidencia y fuerza de la recomendación (según el esquema de la US Preventive Task Force -USPSTF, Guide to Clinical Preventive Services, 1996). La clasificación se describe en la tabla III.

Las intervenciones que en nuestro caso nos interesan (por tener que aplicarlas) son:

\begin{tabular}{|l|l|}
\hline \multicolumn{2}{|l|}{ Tabla III. Niveles de evidencia y Fuerza de la recomendación } \\
\hline Calidad de la evidencia \\
\hline I & $\begin{array}{l}\text { Evidencia obtenida al menos de un ensayo clinico controlado aleatorizado de } \\
\text { manera apropiada }\end{array}$ \\
\hline II- 1 & Evidencia obtenida de ensayos clinicos controlado bien disenados sin aleatorización \\
\hline II- 2 & $\begin{array}{l}\text { Evidencia obtenida de estudios analiticos bien disenados de poblaciones o casos y } \\
\text { controles con preferencia de más de un centro o grupo de investigación }\end{array}$ \\
\hline II- 3 & Evidencia obtenida de series temporales múltiples con intervención o sin ella \\
\hline III & $\begin{array}{l}\text { Opiniones de autoridades respetadas, basadas en la experiencia clinica, estudios } \\
\text { descriptivos e informes de casos, o informes de comités de expertos }\end{array}$ \\
\hline Fuerza de la recomendación \\
\hline A & Existen evidencias sólidas para apoyar la inclusión de la actividad \\
\hline B & Existen suficientes evidencias para apoyar la inclusión de la actividad \\
\hline C & $\begin{array}{l}\text { No existen evidencias suficientes para recomendar o rechazar la inclusión de la } \\
\text { actividad, pero es posible hacer recomendaciones apoyándose en otros motivos }\end{array}$ \\
\hline D & Existen suficientes evidencias para rechazar la inclusión de la actividad \\
\hline E & Existen evidencias sólidas para apoyar rechazar la inclusión de la actividad \\
\hline
\end{tabular}

a) En cuanto a la detección del consumo en una persona de riesgo:

- Garantizar la privacidad: nivel de evidencia I, Fuerza A.

- Captación oportunista: nivel de evidencia I, Fuerza A.

b) En cuanto a la rehabilitación (deshabituación):

- Revisar tratamientos previos: Nivel de evidencia III, Fuerza B (se echa de menos la revisión de recaídas previas y las causas asociadas).

- Comunicación basada en la empatía: Nivel de evidencia I, Fuerza A.

- Refuerzo motivacional (en nuestro caso la recuperación laboral): Nivel de evidencia I, Fuerza A.

- Coordinación entre todos los recursos del sistema: Nivel de evidencia I, Fuerza A.

c) En cuanto al control evolutivo:

- Establecer consultas programadas para identificar problemas que incrementan el riesgo de recaída: Nivel de evidencia II, Fuerza A (en el caso del seguimiento en los SPRL -en el mismo lugar de trabajo- las consultas no son programadas sino que aleatoriamente se llama al trabajador en los momentos menos esperados, así el trabajador no tiene tiempo de "prepararse" para la consulta).

- Monitorización del uso de sustancias: nivel de evidencia III, Fuerza B (igual que en el caso anterior, los SPRL tienen la ventaja de 
estar en el mismo lugar y tiempo que el trabajador en fase de deshabituación, una ventaja con la que no cuenta el médico de familia).

Si bien existen evidencias sólidas sobre diversas actividades a realizar en la intervención en drogas desde Atención Primaria, desde la Medicina del Trabajo algunas de estas intervenciones (en concreto en la fase de rehabilitación) no sólo se pueden realizar de la misma manera, sino que las condiciones de accesibilidad al paciente (trabajador) hace que el éxito de las mismas esté prácticamente garantizado.

\section{OBSERVACIONES FINALES}

Si el SPRL aborda el seguimiento de la reincorporación laboral de un trabajador sanitario con "alta" laboral y aún en tratamiento de deshabituación:

1. ¿Durante cuanto tiempo?, ¿más de dos años?. Sí, al menos dos años.

2. Se genera un alto gasto de recursos -personales y materiales-, tanto del propio SPRL, como del Centro Sanitario (autorización de gastos de analíticas toxicológicas, gastos de envío de muestras al laboratorio toxicológica). Para evitar el desgaste de los profesionales que colaboran en el seguimiento (médico del trabajo, responsables inmediatos del trabajador en fase de rehabilitación, compañeros -que tienen que asumir mayor carga de trabajo en tanto que el afectado tiene limitadas algunas tareas, como p.ej. guardias-) es imprescindible tener un procedimiento de actuación al cual ceñirse.

3. Existe la posibilidad de obtener falsos negativos pese al cuidado extremo del procedimien- to (no se pueden garantizar la detección de consumos esporádicos, ni siquiera con el análisis de pelo).

4. Se debe hacer una previsión de recaídas según las condiciones y los riesgos individuales (contemplar esa posibilidad en el "informe de aptitud" al final del proceso).

Pero si el SPRL no aborda dicho seguimiento: .El trabajador "sanitario enfermo" pierde su única vía de recuperación.

Por eso es fundamental la implicación de todos los profesionales que intervienen en el procedimiento (SPRL, política de prevención de las propias Direcciones de las áreas sanitarias, PAIPSE), con honestidad y sentido común. Y, como dicen los seguidores de un famoso equipo -de fútbol, en este caso-, "You'll never walk alone" (a quien corresponda) (figura 5).

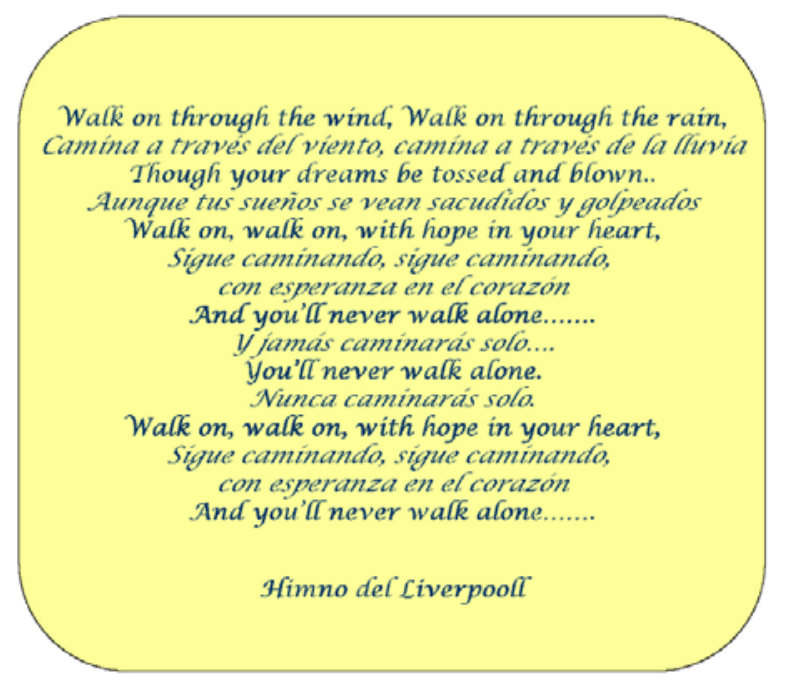

Figura 5. 


\section{BIBLIOGRAFIA}

1. Otero Dorrego C, Huerta Camarero C, Duro Perales N. Drogodependencias en personal sanitario, una visión desde la medicina del trabajo (I). Aspectos jurídico-legales y datos epidemiológicos. Med Segur Trab 2008; 54(211):15-23.

2. Ley 5/2002 de 27 de junio, de Drogodependencias y otros trastornos adictivos (BOCM n ${ }^{\circ} 160$, 8-jul-2002 y BOE n 176, 24-jul-2002).

3. Ley 41/2002, de 14 de noviembre, básica reguladora de la autonomía del paciente y de derechos y obligaciones en materia de información y documentación clínica (BOE nº 274, 15-Nov-2002).

4. Ley Orgánica 3/1986, de 14 de abril, de medidas especiales en materia de salud publica. $\left(B O E n^{\circ}\right.$ 102, 29-Abr-1986).

5. Código de Ética y Deontología, 1999. OMC. España.

6. Ley 31/1995, de 8 de noviembre, Ley de Prevención de Riesgos Laborales. (BOE $\mathrm{n}^{0}$ 269, 10-Nov-1995).

7. Calabrese G. Fármaco-dependencia en anestesiólogos, un gran problema ocupacional actual. Rev. Col. Anest., June 2006, vol.34, no.2, p.103-111. ISSN 0120-3347.

8. Sánchez Uríz MA. Procedimiento de Vigilancia de la Salud Obligatoria. Publicación Interna, Servicio de Prevención de Riesgos Laborales Área 1 SERMAS. Hospital Virgen de la Torre. Madrid.

9. F. Bandrés. Responsabilidad profesional y laboratorio clínico. Sociedad española de dirección y gestión de los laboratorios clínicos.1999.

10. ORDEN 1436/2006, de 21 de julio, del Consejero de Sanidad y Consumo, por la que se aprueba el Plan de Atención Integral al Profesional Sanitario Enfermo, en el ámbito de las instituciones sanitarias de la Comunidad de Madrid. (BOCM n ${ }^{\circ}$ 181, 1-Ago-2006).

11. Arteman Jané, A. Programas especiales dirigidos al médico enfermo. JANO extra marzo 2004. Vol. LXVI n ${ }^{\circ}$ 1514: 1119-1128.
12. K. Wolf, M. Farrel, J. Marsden, M.G. Monteiro, R. Ali, S. Welch, J. Strang. Revisión de los indicadores biológicos de uso ilegal de drogas, consideraciones prácticas y utilidad clínica. RET, Revista de Toxicomanías. № 28, 2001.

13. G Zapater, MJ Perea, C Carrizosa, B Bravo, LJ Segura. Estudio de 156 casos de accidentes laborales mortales en la Comunidad de Madrid. Análisis de alcohol etílico y otras sustancias psicoactivas. TF-C13. Rev. Toxicolo (2003) 20. XV Congreso Español de Toxicología.

14. A Ortega Pérez. ¿Estaba intoxicado por cocaína este individuo? (y II): estimaciones basadas en los efectos de la droga y en las concentraciones de benzoilecgonina. Cuadernos de Medicina Forense No 33. Julio 2003.

15. Kintz P, Villain M, Dumestre V, Cirimele V. Evidence of addiction by anesthesiologists as documented by hair analysis. Forensic Sci Int. 2005 Oct 4;153(1):81-4.

16. Antonio F. Hernández, Fernando Gil, Antonio Pla. Nuevas perspectivas en el análisis de drogas de abuso para el año 2000. Departamento de Medicina Legal y Servicio de Toxicología. Universidad de Granada. Revista electrónica de Ciencia Penal y Criminología. RECPC 01-03 (1999).

17. Perkins de Piacentino A.M.; Locani O.A.; Lorenzo J.L. Drogas en pelo. Sus alcances y limitaciones (I) y (II). Laboratorio de Toxicología y Química Legal. AMA. Argentina, 2005.

18. Gold MS, Melker RJ, Dennis DM, Morey TE, Bajpai LK, Pomm R, Frost-Pineda K. Fentanyl abuse and dependence: further evidence for second hand exposure hypothesis. J Addict Dis. 2006;25(1):15-21.

19. McAuliffe PF, Gold MS, Bajpai LK, Merves ML, Frost-Pineda K, Pomm R, Goldberger BA, Melker RJ, Cendan JC. Second-hand exposure to aerosolized intravenous anesthetics propofol and fentanyl may cause sensitization and subsequent opiate addiction among anesthesiologists and surgeons. Med Hypotheses. 2006;66(5):874-82. Epub 2006 Jan 23. 
20. Domino KB, Hornbein TF, Polissar NL, Renner G, Johnson J, Alberti S, Hankes L: Risk Factors for Relapse in Health Care Professionals with Substance Use Disorders. JAMA. 2005 Mar 23/30; 293(12):1453-60.

21. Kluger MT, Townend K, Laid Law T. Job satisfaction, stress and burn out in Australian specialist anaesthesiologists. Anaesthesia 2003 Apr; 58 (4): $339-45$.

22. Pelton C, Ikeda RM. The California physicians diversion program's experience with recovering anesthesiologists. Journal of Phsychoactive Drugs.1991;23:427-431.
23. Paris RT, Canavan DI. Physician substance abuse impairment: Anesthesiologists vs other specialties. J Addictive Diseases. 1999;18(2):23-27.

24. Gold MS, Byars JA, Frost-Pineda K. Occupational exposure and addictions for physicians: case studies and theoretical implications. Psychiatr Clin North Am. 2004 Dec;27(4):745-53.

25. Casado Vicente V, Araúzo Palacios D. Intervención en drogas desde atención primaria: revisión de guías clínicas. Adicciones. 2004;16(3):225-234. 